www. revis t a d y o. com

\title{
Inversión versus coste del ciclo de vida de los edificios. Proyecciones energético-económicas
}

Investment versus life cycle cost of buildings. Energy-economic projections

Pablo Aparicio, José Guadix, Luis Onieva

Departamento de Organización Industrial y Gestión de Empresas II. Escuela Técnica Superior de Ingenieros. Universidad de Sevilla. Camino de los Descubrimientos s/n 41092.

pabloaparicio@us.es,guadix@us.es, onieva@us.es

Fecha de recepción: 07-10-2014

Fecha de aceptación: 20-02-2015

Resumen: La inversión en la construcción es un factor limitante cuando se desea mejorar la eficiencia energética, debido a que los constructores prestan mayor atención a la inversión que al coste del ciclo de vida durante la vida útil del edificio. Este artículo presenta una metodología desarrollada que permite mejorar la calificación energética de edificios y su coste del ciclo de vida, en la mayoría de los escenarios económicos, las soluciones reducen la inversión, si se escogen los materiales constructivos óptimos.

Palabras clave: inversión, coste del ciclo de vida, construcción, eficiencia energética, calificación energética.

Abstract (English) Investment in construction is limiting by some factors when it is desired improve energy efficiency, because the builders pay more attention to investment, which the life cycle cost over the lifetime of the building. This paper presents a developed methodology to improve the energy rating of buildings and life cycle cost, in the majority of economic scenarios; the solutions reduce investment, if the optimal construction materials are chosen.

The aim of this study focuses on optimizing the life cycle cost of a building with the lowest consumption of energy demand, with the aim of improving environmental quality and energy. A methodology for optimizing the building envelope or skin of a building and the active elements that compose is set, to study the power consumption and the corresponding saving is established, considering the environmental impact and the cost of it. The application of a heuristic Tabu expedites time to resolution of the problem.

However, the life cycle cost study is necessary to develop projections possible about the economic future of a building. Homeowners should understand the different economic situations in order to protect their investment. Moreover, investment is often a limiting factor in the decisions of construction.

It tends to minimize the investment, within the law when it comes to achieve an energy rating and energy efficiency index, therefore you must know the effect of the investment when optimizing a building. In the future pricing of carbon emissions may provide an incentive for the deployment of efficient and low-carbon technologies across Europe.

Those responsible of strategic investments in buildings often make decisions more or less intuitively, generally based on experience. The study of a building for different economic projections, we propose and consider different climatic zones. The building of residential character is described in detail. The application of the methodology presented in this paper can offer the user the study of buildings to various economic situations.

The paper presents 956 case studies of this building. By studying Spanish 12 climatic zones, different economic scenarios are presented. The results show that the application of the methodology allows a reduction of $\mathrm{CO} 2$ emissions, reduced demand for heating, and the investment is reduced in a considerable number of life cycle cost cases.

The final decision is applied by the responsible of making the decision, although the data obtained by applying this methodology are used to make that decision. The methodology provides a competitive advantage when the building is presented. Given the uncertainties in the market, it should not make overly optimistic assessment, although the system helps decision making, the experience will be key to decide the best economic scenario.

Keywords: investment, life cycle cost, construction, energy efficiency, energy rating. 


\section{Introducción}

En 2012 se presenta una metodología para la optimización de la demanda y el estudio del coste del ciclo de vida de los edificios (Aparicio et al., 2012). El objetivo de este estudio se centra en optimizar el coste del ciclo de vida (CCV) (Zabalza et al, 2013) de un edificio con el menor consumo de demanda energética, con la pretensión de mejorar la calidad ambiental y energética. Se establece una metodología de optimización de la envolvente o epidermis de un edificio y de los elementos activos que la conforman, para estudiar el consumo energético y el ahorro correspondiente, considerando el impacto medioambiental y el coste de éste. La aplicación de una heurística tabú (Glass and Fu, 2013) permite agilizar el tiempo de resolución del problema.

Sin embargo, el estudio del CCV hace necesario desarrollar posibles proyecciones sobre el futuro económico de un edificio. Los propietarios deben conocer las diferentes situaciones económicas a las que se puede enfrentar su inversión. Por otra parte, a menudo la inversión es un factor limitante en las decisiones de la construcción (Mata et al., 2015; Monetti et al., 2015; Markandya, 2014, 2015). Se tiende a minimizar la inversión, dentro de la legalidad a la hora de alcanzar una calificación energética (Andaloro et al., 2010) e índice de eficiencia energética (IEE), por tanto, se debe conocer el efecto de la inversión cuando se busca la optimización de un edificio.

En la actualidad, la calificación se expresa mediante una letra, desde la letra A, para los edificios más eficientes, a la letra $\mathrm{G}$ para los menos eficientes y está se relaciona con el IEE que define el baremo para la clasificación de los inmueble y se expresa en valores de kilogramos de dióxido de carbono por metro cuadrado emitidos (kg CO2/m2).

En el futuro se aplicará una tarificación de las emisiones de carbono (Corporate Leaders Group, 2012) que puede constituir un incentivo para el despliegue de tecnologías eficientes y con baja emisión de carbono en toda Europa. Estas iniciativas fomentarán el interés por el consumo energético en la compra de vivienda y la mejora de la eficiencia energética.

La eficiencia energética, es el conjunto de acciones que permiten optimizar la relación entre la cantidad de energía consumida y los productos-servicios finales obtenidos, es el término fundamental que permite medir la sostenibilidad de una obra de arquitectónica. Ésta refleja la energía que requiere un edificio para su funcionamiento, sin embargo el coste energético total de un edificio es mucho mayor y está relacionado con su ciclo de vida. El ciclo de vida de un edificio comprende todas las tareas que son necesarias para su producción, uso y reutilización del terreno, es decir la demolición del edificio. La metodología aplicada se basa en el estudio del CCV durante el uso o vida útil del edificio.

La energía es un sector estratégico en el desarrollo económico, y se ha convertido en una preocupación primordial tras la crisis de la década de 1970. El temor a nuevas situaciones similares y los consecuentes impactos en las economías globales, han estimulado la investigación económica de la energía.

Las últimas proyecciones de las necesidades mundiales de energía por parte de la Energy Information Administration (EIA, 2013) para el año 2040 indican un crecimiento del $54,6 \%$ en el consumo de energía en comparación con 2010. Los combustibles fósiles (petróleo, gas natural y carbón) se pronostica que continuarán dominando el mix energético, proporcionando $78,5 \%$ de la energía que el mundo necesita, en comparación con $84,1 \%$ en 2010 . Las energías renovables se espera que crezcan de $10,7 \%$ en 2010 al $14,5 \%$ en 2040. Sin embargo, los objetivos de descarbonización de la Unión Europea (UE) son bien distintos, la UE se comprometió a reducir las emisiones de gases de efecto invernadero entre un $80 \%$ y un $95 \%$ por debajo de los niveles de 1990 para 2050 (UE, 2011).

El aumento de la oferta de combustibles fósiles ayuda a proporcionar más tiempo para la investigación y el desarrollo necesarios para la próxima transición energética, si bien este crecimiento finalizará a corto plazo. El crecimiento económico sostenible va a requerir nuevas inversiones públicas y privadas que mejoren la energética tanto de los materiales (COM, 2011) como de los sistemas y otros elementos, que el (Ministerio de Fomento, 2014) recoge el requerimiento de un análisis de la rentabilidad de los escenarios estratégicos, y estima en el caso de la rehabilitación de viviendas, para la cual también es aplicable esta metodología, "para multiplicar las potencialidades de la rehabilitación sería 
deseable contar con un marco de financiación adecuado, que contemple tipos de interés reducido y plazos largos. Se consideran condiciones suficientemente atractivas para los propietarios los créditos a 20 años y a un interés del $5 \%$ ".
En la Tabla 1, se presenta un posible escenario económico. El crecimiento anual del precio de los recursos energéticos y del consumo de los edificios (EIA, 2013), donde el aumento de la temperatura global pronostica una reducción de la calefacción y un aumento de la refrigeración.

\begin{tabular}{|c|c|c|c|c|c|c|c|}
\hline \multirow[t]{2}{*}{ Recurso } & \multicolumn{6}{|c|}{ Casos de referencia } & \multirow{2}{*}{$\begin{array}{l}\text { Crecimiento anua } \\
\text { 2011-2040 (\%) }\end{array}$} \\
\hline & 2011 & 2020 & 2025 & 2030 & 2035 & 2040 & \\
\hline Propano & 25,06 & 23,41 & 24,7 & 25,73 & 26,70 & 27,99 & $0,4 \%$ \\
\hline Gasolina & 26,38 & 26,91 & 29,08 & 31,26 & 33,71 & 36,54 & $1,1 \%$ \\
\hline Gas natural & 10,80 & 11,78 & 12,67 & 13,37 & 14,60 & 16,36 & $1,4 \%$ \\
\hline Electricidad & 34,34 & 33,62 & 33,96 & 34,56 & 35,42 & 37,10 & $0,3 \%$ \\
\hline \multicolumn{8}{|c|}{ Total de energía consumida } \\
\hline Calefacción & 5,33 & 5,05 & 4,93 & 4,83 & 4,71 & 4,57 & $-0,5 \%$ \\
\hline Refrigeración & 2,88 & 2,86 & 3,10 & 3,35 & 3,60 & 3,84 & $1 \%$ \\
\hline
\end{tabular}

En el sector residencial, la iniciativa "Ciudades inteligentes" (Smart Cities) (IDAE, 2012) de la UE tiene como objetivo lograr una mayor eficiencia en el sector. En este contexto se tienen dos objetivos: mejorar la eficiencia en el uso final y, desarrollar y aumentar la proporción de energías renovables sin empeorar el nivel de vida de los ocupantes. La demanda de energía de un hogar se puede reducir entorno a un $30 \%$.

\section{Metodología}

La simulación permite la evaluación y estudio de los objetivos y aspectos de diseño previos a la construcción. Asiste en el estudio e identificación de soluciones y alternativas. Permite realizar un análisis de sensibilidad para visualizar de forma inmediata las ventajas y desventajas económicas de una modificación del proyecto, mediante la evaluación y ponderación de las alternativas respecto de los objetivos o criterios globales.

En el estudio de Aparicio et al. (2012) se establece una metodología para ayudar en la toma de decisiones a los arquitectos, en esta se aplican los cálculos inicial y final mediante la herramienta para el cálculo de la demanda energética de un edificio (LIDER) que aplica la Directiva 2002/91/CE. El proceso de cálculo con esta herramienta suele ser lento, aunque riguroso, por lo que se ha aplicado la metaheurísticas Búsqueda Tabú (Glover y Laguna, 1997) que incorpora los modelos simplificados de demanda energética desarrollados por (Álvarez et al., 2012) para agilizar el análisis de forma exitosa.

La toma de decisiones tiene que unir factores ambientales (emisiones $\mathrm{CO} 2$ ), energéticos (demanda), financieros (inversión, costes emisiones $\mathrm{CO} 2$ y demanda) que comprenden el necesario análisis para la toma de decisiones, a continuación se presenta el análisis económico aplicado.

\subsection{Análisis económico}

El CCV durante la vida útil de un edificio es un método de análisis económico de todos los costes relacionados con la construcción, operación y mantenimiento de un proyecto durante un periodo de tiempo definido. En el análisis se asumen tasas escaladas para dar cuenta de los aumentos en los costes a través del tiempo. Cuando se analiza económicamente un edificio, la solución depende de los pesos relativos entre los diferentes criterios de optimización, siendo uno de ellos, el número de años considerados en el estudio de rentabilidad económica, el coste de la energía, el tipo de interés del dinero utilizado para el cálculo del Valor 
Actual Neto (VAN) y el punto inicial elegido.

El procedimiento de obtención de CCV tiene en cuenta los siguientes costes:

- El coste de inversión, de los elementos constructivos: la transmitancia térmica de los cerramientos (muros, cubiertas y suelos); la transmitancia térmica, el factor solar y la permeabilidad de ventanas; las transmitancias térmicas lineales de puentes térmicos; la permeabilidad y el control de la ventilación.

- El coste de operación debido al consumo energético de las demandas de calefacción y refrigeración del edificio estudiado.

- El coste por emisiones de $\mathrm{CO} 2$ generado por esas demandas.
El cálculo del CCV es la suma del coste de inversión, más el de operación, más el coste por emisiones de $\mathrm{CO} 2$, que se produce durante los años de vida del edificio.

Como indica el suplemento de la Directiva 2010/31/UE (16.1.2012), en los estados miembros se efectuará un análisis para determinar la sensibilidad de los resultados tras el cálculo de los cambios en los parámetros aplicados, cubriendo al menos el impacto de la diferente evolución de los precios energéticos y de las tasas de descuento para los cálculos macroeconómicos y financieros, durante un periodo de 30 años, a ser posible también otros parámetros que se espera que tengan un impacto significativo sobre el resultado de los cálculos, como por ejemplo la evolución de los precios de la energía. Dada la incertidumbre en la evolución del tipo de interés del dinero y del precio de la electricidad, se pueden realizar estudios de los diferentes escenarios económicos.

$$
C C V(€)=\text { Inversión }\left(\text { Consumo }_{\text {calefaccion año } 1}+\text { Coste }_{\text {emisiones año } 1}\right) \bullet \text { Anualidad }
$$

Donde :

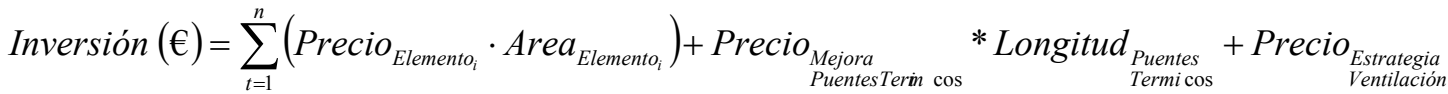

$$
\begin{aligned}
& \text { Consumo }_{\text {total calefacción }}(€ / \text { año })=\sum_{t=1}^{n}\left(\text { Consumo }_{\text {calefacción añot }}(€ / \text { año })\right) \\
& \text { Coste }_{\text {total emisiones }}(€ / \text { año })=\sum_{t=1}^{n}\left(\text { Coste }_{\text {emisiones }} \text { año } t(€ / \text { año })\right) \\
& \text { Anualidad }(\text { años })=\sum_{t=1}^{n} \frac{\left(1+k_{1}\right)^{t}}{(1+k)^{t}} \\
& \text { Consumo }_{\text {calefacción cñot }}(€ / \text { año })=\frac{\text { Demanda }_{\text {calefacción añot }}\left(\mathrm{kWh} / \mathrm{m}^{2} \text { año }\right)}{\text { Rendimiento }_{\text {sistema calefacción }}} \cdot \operatorname{Precio}_{\text {energia añot }}(€ / \mathrm{kWh})
\end{aligned}
$$

En esta metodología son conocidos los precios de cada elemento constructivo, de la mejora de puentes térmicos, de las estrategias de ventilación, el coste por emisiones de CO2 (20€/tonelada, límite inferior (Kwiatkowski, 2013)), y el precio de la energía usada $(0,13 € /$ kWh de electricidad: tarifa estándar sin discriminación horaria) (BOE, 2014).

Por su parte, el rendimiento medio estacional del sistema de calefacción, puede suponerse constante y conocido, esto permite obtener el consumo de energía final. El cálculo de la demanda de calefacción se realiza en base a la definición de (Aparicio et al., 2012).

\section{Caso de estudio}

Se plantea el estudio de un edificio para diferentes proyecciones económicas y se contemplan diferentes zonas climáticas (Salmerón et al., 2013). El edificio de carácter residencial (Figura 1), con 164,4 m2 de área acondicionada, dividido en dos plantas y un sótano, con una cubierta a dos aguas. La altura media de las plantas es de $2,5 \mathrm{~m}$. El área de transferencia es de $209,9 \mathrm{~m} 2$. El área de la cubierta es de $63,56 \mathrm{~m} 2$, el área de suelos es de $63,57 \mathrm{~m} 2$, el área de muros es de $62,81 \mathrm{~m} 2$, el área de ventanas es de 19,91 m2.

Actualmente, el interés de las obligaciones españolas a 30 años está en el 4,519\% (Marzo 2014). Mientras que las tendencias y las previsiones a largo plazo sobre el desarrollo económico y crecimiento del producto 
interior bruto (PIB) están en el 1,9 \% anual (PwC Economics, 2013). Si observamos la evolución de los mercados minoristas de electricidad en el período 20082012, casi todos los Estados miembros de la UE han visto un aumento en los precios de electricidad de sus hogares. En promedio, los precios de la electricidad de los hogares de la UE se incrementaron en más de un
3\% al año, mientras otros países como España han experimentado incrementos anuales promedio de 9-10\%. Para el análisis, se consideraron diversos escenarios, con intereses de obligaciones a 30 años entre 0 y $10 \%$, y con precios de la energía más bajos $(-0,04 \%)$ y más altos (10\%).

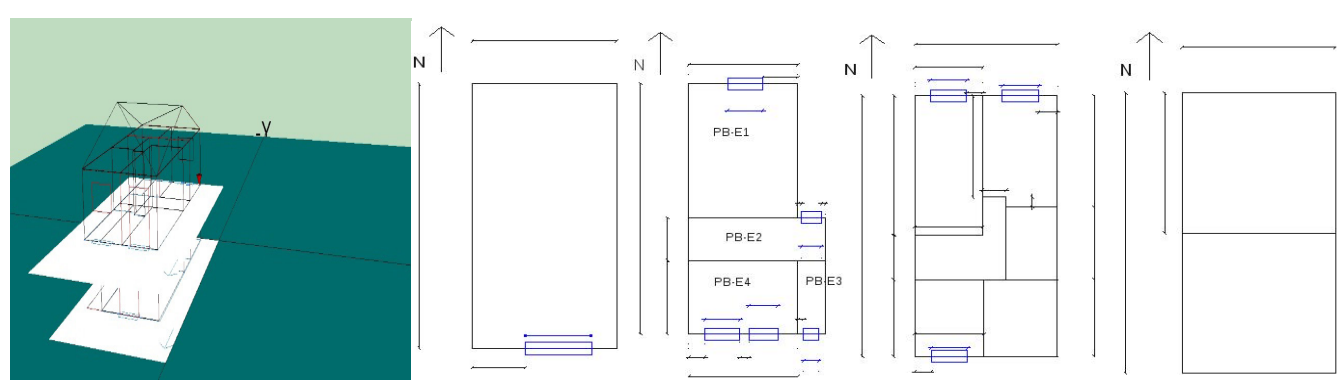

Figura 1 Edificio de estudio.

\section{Resultados}

La aplicación de la metodología al problema presentado en el apartado 3, en la búsqueda de una solución óptima en CCV (Figura 2, gráfica superior), no supone un aumento de la inversión inicial, como se puede observar en el proceso de la solución presentada en la Figura 2 (gráfica inferior) donde sólo son tenidos en cuenta los costes de los elementos constructivos de la envolvente del edificio.

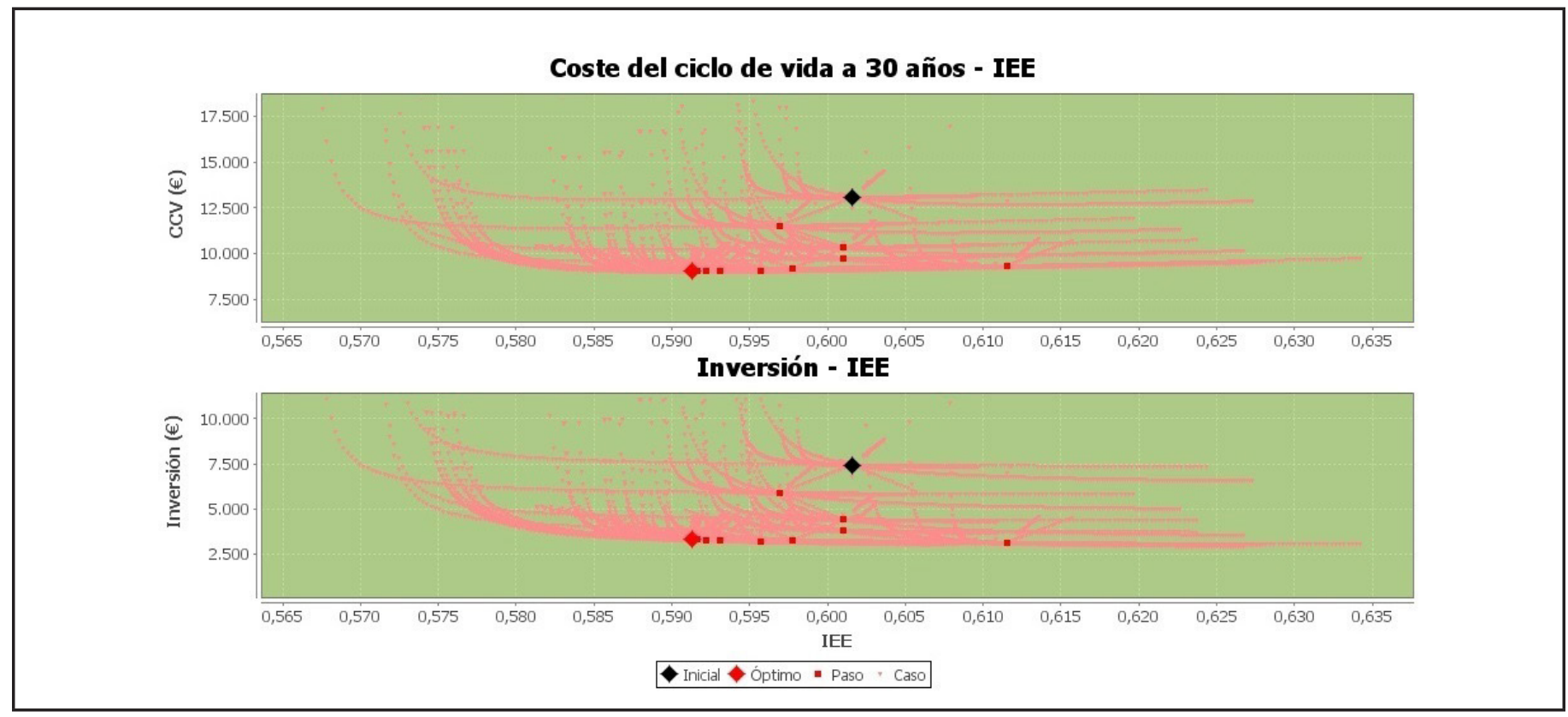

Figura 2 Representación de un caso de estudio. CCV (Superior) e inversión (Inferior) frente al IEE para un interés del $4 \%$ e incrementos del precio de la energía del $3 \%$. Estudio a 30 años.

La aplicación de la metodología para las distintas zonas climáticas se presenta en la Figura 3. Se decide mostrar los datos para un interés del dinero del $4 \%$ e incrementos del precio de la energía del $3 \%$ en estudios a 30 años como nos recomienda la UE en la Directive 2010/31/EU.

Tras la aplicación de 956 casos, mediante el estudio de las 12 zonas climáticas españolas. En España, para la lim- itación de la demanda energética se establecen 12 zonas climáticas identificadas mediante una letra, correspondiente a la división de invierno $(A, B, C, D, E)$, y un número $(1,2,3,4)$, correspondiente a la división de verano. Surgen 12 zonas climáticas debido a que se excluyen las combinaciones imposibles para la climatología española, en la figura 3 y 4 se definen las 12 posibles zonas (BOE, 2013). Donde la A representa el 
invierno más suave, y el 1 representa el verano más suave. Estas zonas climáticas se presentan frente a los diferentes escenarios económicos mediante variaciones en el interés del dinero y precio de la energía. De estas variaciones, se observa que la aplicación de la metodología permite una reducción de las emisiones de CO2 de un 29,28\%. Para la demanda de calefacción hay una reducción media del $42,9 \%$, mientras en la demanda de refrigeración la reducción media es casi nula. El CCV se reduce en un $17,8 \%$ mientras que la inversión media se reduce en un $32,3 \%$, si bien, en el $16,7 \%$ de los casos la inversión inicial se incrementó.
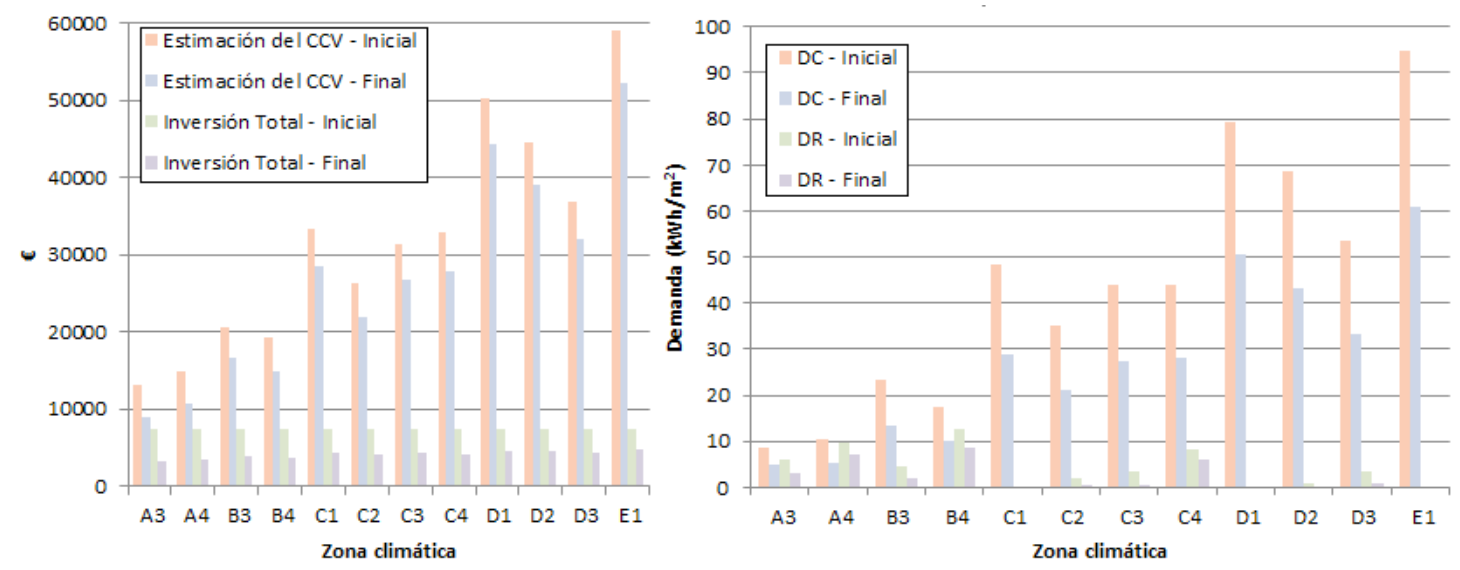

Figura 3 Estimación del CCV e inversión (izquierda) y estimación de la demanda de calefacción (DR) y refrigeración (DR) (derecha) de un edificio situado en diferentes zonas climáticas para un interés del dinero del $4 \%$ e incrementos del precio de la energía del $3 \%$. Estudio a 30 años.
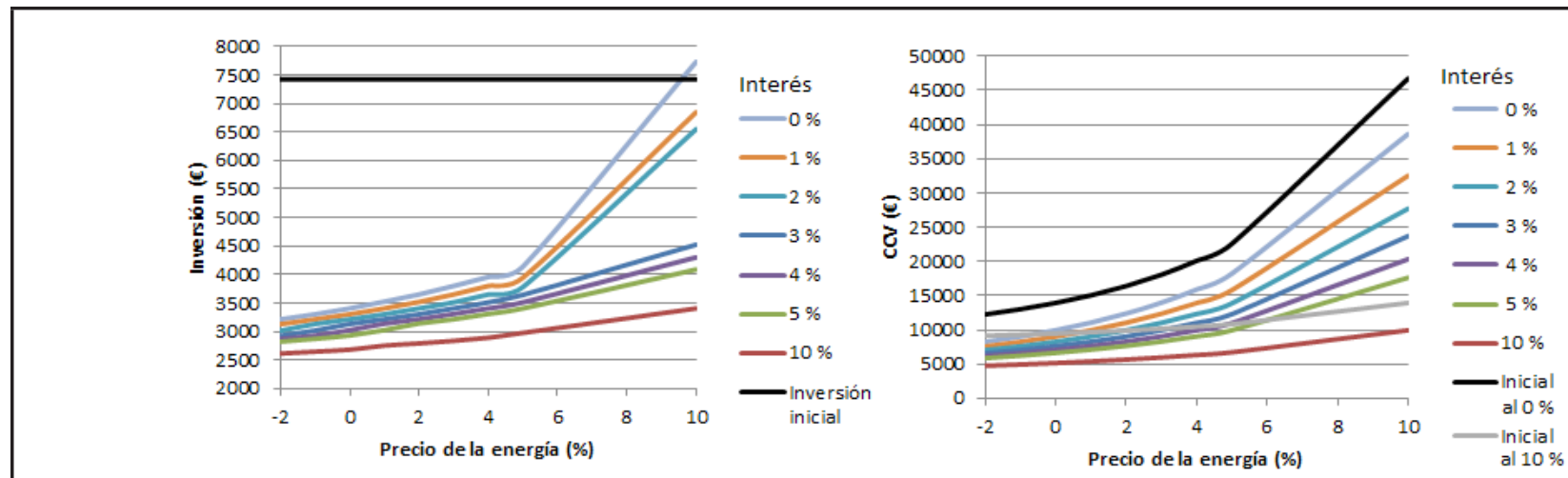

Figura 4 Escenarios económicos de la inversión y del CCV de un edificio en base a las variaciones del interés del dinero y del precio de la energía, para un estudio a 30 años. En base a soluciones óptimas en el IEE-CCV para un edificio en la zona climática A3.

En la Figura 4, se presentan diferentes soluciones finales tras aplicar la metodología al edificio de estudio, se ha elegido la zona climática $\mathrm{A} 3$, correspondiente a la ciudad de Málaga y cuyo clima es suave, no obstante la metodología permite mejorar la demanda y CCV de cualquier zona climática. Para el ejemplo propuesto, la solución inicial obtenida a interés nulo, partiría de un edificio con calificación energética $C$ y se obtuvo un edificio de calificación energética $B$. Los elementos constructivos modificados sería, una cubierta cuyo valor de $\mathrm{U}$ inicial es $0.57 \mathrm{~W} / \mathrm{m} 2 / \mathrm{K}$ a $0.29 \mathrm{~W} / \mathrm{m} 2 / \mathrm{K}$, un suelo cuyo valor $U$ inicial es $0.23 \mathrm{~W} / \mathrm{m} 2 / \mathrm{K}$ a $0.51 \mathrm{~W} / \mathrm{m} 2 / \mathrm{K}$, en el caso de las paredes de $0.49 \mathrm{~W} / \mathrm{m} 2 / \mathrm{K}$ a $0.41 \mathrm{~W} /$ $\mathrm{m} 2 / \mathrm{K}$, en el caso de la ventana norte por ejemplo, tenía un valor $U$ de $3.3 \mathrm{~W} / \mathrm{m} 2 / \mathrm{K}$, g de 0.75 y p de $27.0 \mathrm{~m} 3 /$
hm2@100Pa y se cambió por una ventana de $U$ de $1.8 \mathrm{~W} / \mathrm{m} 2 / \mathrm{K}, \mathrm{g}$ de 0.70 y p de 50.0 m3/hm2@100Pa, también se produjeron cambios en el resto de ventanas. En (Aparicio, 2012) se pueden encontrar expresados resultados de la metodología para los elementos constructivos. En los resultados mostrados en este documento, se observa que se consiguen cambios de calificación energética y ahorro en inversión, como se puede ver en la figura 4.

En la figura se presentan diferentes escenarios económicos de un edificio en base a las variaciones del interés del dinero y del precio de la energía, para los cuales la reducción de la estimación del CCV es del $29,07 \%$ y del $50,39 \%$ en la inversión. En la solución 
optimizada, la menor reducción del CCV es del 16,56\% frente a la mayor del $48,01 \%$, en el caso de la inversión existe un aumento máximo del $14,87 \%$, mientras que la mayor reducción es del $64,75 \%$.

En el estudio realizado, se observó que para 160 casos el aumento medio de cada euro de inversión generó un ahorro de $0,48 €$ al año respecto al CCV inicial. Por otra parte, en los 796 casos restantes, se redujo la inversión, y cada euro reducido generó una reducción de $0,07 €$ al año respecto al CCV inicial del edificio.

Tras los resultados, se observa que la metodología podría ayudar a los constructores a fomentar el diseño arquitectónico optimizando el CCV de un edificio. Se destaca que si se seleccionan los elementos constructivos adecuados, optimizar el CCV y mejorar la eficiencia energética no es sinónimo de mayor inversión.

Como se pudo observar en la Tabla 1 anterior, el cambio climático previsiblemente puede variar las tendencias en el consumo, se estima un crecimiento anual del $1 \%$ en la demanda de calefacción (periodo 20112040), y una reducción de la calefacción del 0,5\%. Estos datos plantean la posibilidad de realizar estudios del CCV que incluyan diferentes proyecciones de la climatología.

\section{Conclusiones}

Las decisiones que toman los responsables de las inversiones estratégicas de las construcciones se suelen tomar de forma más o menos intuitiva, en base a la propia experiencia. La aplicación de la metodología presentada permite el estudio de los edificios frente a diversas situaciones económicas, mediante el estudio del coste del ciclo de vida de los edificios.

En este trabajo se ha plasmado de forma concreta un caso de estudio para un edificio, a partir de los datos obtenidos por esta metodología, aun así, las decisiones se deja en manos del responsable final del diseño del edificio, el cual, ante las incertidumbres en el mercado, conviene que no realice una valoración excesivamente optimista. Si bien el sistema ayuda a la toma de decisiones, la experiencia será clave en la decisión sobre el escenario económico en base al cual tomar la decisión.

La metodología ofrece una ventaja competitiva a la hora de presentar el edificio, el uso de esta metodología permitiría a los arquitectos y a los constructores ofrecer las proyecciones de coste del edificio en base a la demanda energética para la que fue diseñado. Rechazar proyectos de riesgo o la posibilidad de elegir o comparar posibles diseños, no solo por sus materiales o su calificación energética sino por el coste esperado durante la vida útil del edificio.

\section{Referencias}

ANDALORO, A., SALOMONE, R., IOPPOLO, G., ANDALORO, L. (2010). «Energy certification of buildings: A comparative analysis of progress towards implementation in European countries». Energy Policy, 38(10), pp. 5840-5866, ISSN 0301-4215, http:// dx.doi.org/10.1016/j.enpol.2010.05.039.

ÁLVAREZ, S., MOLINA, J., SÁNCHEZ, F., SALMERÓN, J. (2012). "Guía IDAE: Manual de fundamentos técnicos de calificación energética de edificios existentes CE3». Madrid.

APARICIO, P., GUADIX, J., ONIEVA, L., RODRÍGUEZ, M. (2012). «Optimización de la demanda de calefacción y análisis del coste del ciclo de vida de los edificios». Dirección y Organización, 48(1), pp. 23-28.

BOE (2013). «Orden FOM/1635/2013, de 10 de septiembre, por la que se actualiza el Documento Básico DB-HE "Ahorro de Energía", del Código Técnico de la Edificación» 219, de 12 de septiembre de 2013, pp. 67137-67209.

BOE (2014). «Orden IET/2444/2014, de 19 de diciembre, por la que se determinan los peajes de acceso de energía eléctrica para 2015» núm. 312, de 26 de diciembre de 2014, pp. 105486-105504.

COM (2011). «Commission staff working paper. Materials Roadmap Enabling Low Carbon Energy Technologies». Brussels, 13.12.2011 SEC (2011) 1609 final

CORPORATE LEADERS GROUP AND THE UNIVERSITY OF CAMBRIDGE (2012). The carbon price communiqué 2012. URL: http://www.climatecommuniques.com/

ENERGY INFORMATION ADMINISTRATION, EIA (2013). U.S. Annual Energy Outlook 2013.

GASS, S.I., FU, M.C. (2013). «Encyclopedia of Operations Research and Management Science», DOI 10.1007/978-1-4419-1153-7, Springer Science+Business Media New York 2013. pp.15371544.

GLOVER, F., LAGUNA, M. (1997). TabuSearch. Kluwer Academic Publishers. 
HARRIS, J., ANDERSON, J., SHAFRON, W. (2000). «Investment in energy efficiency: a survey of Australian firms», Energy Policy, 28 (12), 1 October 2000, pp. 867-876, ISSN 0301-4215, http://dx.doi. org/10.1016/S0301-4215(00)00075-6.

IBERDROLA (2008). Información de tarifas eléctricas y modalidades de contratación 2008. Disponible vía https://www.iberdrola.es/02sica/gc/es/doc/triptico_ tarifas_elect_08.pdf

IDAE (2012). «Mapa tecnológico ciudades inteligentes "Smart Cities"», Instituto para la Diversificación y Ahorro de la Energía, (Borrador. Abril 2012)

KWIATKOWSKI, J., PANEK, A., LEHMDEN, A., UNGER, J. (2013). «Consideration about the cost optimal methodology to determine energy performance requirements for buildings. Decision-support tools and assessment methods». Central Europe towards Sustainable Building 2013.

MARKANDYA, A., LABANDEIRA, X., RAMOS, A. (2015). «Policy instruments to foster energy efficiency», Green Energy and Efficiency (pp. 93-110). Springer International Publishing.

MARKANDYA, A., LABANDEIRA, X., RAMOS, A. (2014). «Policy Instruments to Foster Energy Efficiency», WP01/2014, www.eforenergy.org. ISSN nº $2172 / 843$

MATA, É. MEDINA, G., SASIC, A., JOHNSSON, F. (2014). «Modelling opportunities and costs associated with energy conservation in the Spanish building stock», Energy and Buildings, 88, pp. 347-360, ISSN 0378-7788, http://dx.doi.org/10.1016/j.enbuild.2014.12.010.
MINISTERIO DE FOMENTO, GOBIERNO DE ESPAÑA (2014). «Estrategia a largo plazo para la rehabilitación energética en el sector de la edificación en España en desarrollo del artículo 4 de la directiva 2012/27/UE». Secretaría de Estado de Infraestructuras, Transporte y Vivienda.

MONETTI, V., FABRIZIO, E., FILIPPI, M. (2015). «Impact of low investment strategies for space heating control: Application of thermostatic radiators valves to an old residential building"», Energy and Buildings, ISSN 0378-7788, http://dx.doi.org/10.1016/j. enbuild.2015.01.001.

PWC ECONOMICS (2013). The world in 2050. The BRICs and beyond: prospects, challenges and opportunitie. PricewaterhouseCoopers LLP.

SALMERÓN, J., ÁlVAREZ, S., MOLINA, J., RUIZ, A., SÁNCHEZ, F. (2013). "Tightening the energy consumptions of buildings depending on their typology and on climate severity indexes». Energy and Buildings, 58(1), pp. 372-377.

UNIÓN EUROPEA, UE (2011). «Hoja de Ruta de la Energía para 2050». Bruselas.

ZABALZA, I., ARANDA, A., SCARPELLINI, S. (2009). «Life cycle assessment in buildings: State-of-the-art and simplified LCA methodology as a complement for building certification». Building and Environment, 44(12), pp. 2510-2520, ISSN 0360-1323, http://dx. doi.org/10.1016/j.buildenv.2009.05.001. 